\title{
THE EULER CHARACTERISTIC AND INEQUALITIES FOR KLEINIAN GROUPS
}

\author{
WILLIAM ABIKOFF ${ }^{1}$
}

\begin{abstract}
A class of torsion-free finitely generated Kleinian groups is defined. For groups $G$ in this class, sharp versions-with error terms-of standard inequalities are given. Also, sharp estimates are given for the number of cusps and the sum of the ranks of maximal nonconjugate cyclic parabolic subgroups.
\end{abstract}

Most of the standard results in the theory of Kleinian groups are statements about the borders of hyperbolic 3-manifolds-at least when the groups are torsionfree. This observation is essentially due to Poincaré, although the systematic study has been left to more recent times. The theory of sufficiently large 3-manifolds was used by Marden [6] to show that the algebraically indecomposable groups have a great deal of geometric structure. Great strides in understanding the structure of these manifolds have been taken by Thurston, but most of these results have yet to appear. In this paper we will use an alternate hypothesis, conceivably, but not obviously, more general than that of Marden, to give geometric meaning to the Euler characteristic of a Kleinian group. Once that is accomplished, many of the classical inequalities in Kleinian group theory will appear in a sharp form. The major new ingredient in these inequalities is $\beta_{2}(N)$, the second Betti number of an ample submanifold of the 3-manifold of the Kleinian group. For groups satisfying our hypothesis, estimates due to Bers [3], Ahlfors [2], Marden [6] and Sullivan [9] are refined. The major new inequality is the bound on the sum of the ranks of nonconjugate primary parabolic conjugacy classes in a finitely generated Kleinian group in terms of the number of generators of the group. The bound improves the one given by Sullivan (with clarifications by Kra [5]) and is cited in his paper.

The paper is organized as follows: The first two sections give preliminary definitions and necessary results from 3-manifold theory. $\S 3$ gives a deficiency index for Kleinian groups with a well-positioned ample submanifold. The deficiency index is used in the succeeding two sections to derive the inequalities. In the last section, the validity of the main topological hypothesis, namely the existence of a well-positioned ample submanifold, is discussed.

During the course of the study described herein, the author immensely profited from conversations with R. Craggs, P. Shalen and G. A. Swarup.

1. Preliminaries. Let $G$ be a finitely generated discrete group of orientation preserving isometries of the 3-dimensional hyperbolic space $\not^{3}$. $G$ is called a Kleinian group. $G$ is called elementary if $G$ has an abelian subgroup of finite index.

Received by the editors July 23, 1984 and, in revised form, June 12, 1985.

1980 Mathematics Subject Classification. Primary 30F40, 57N10.

${ }^{1}$ Research partially supported by the National Science Foundation. 
We shall assume throughout this paper that $G$ is nonelementary. We shall further assume that $G$ acts on $\mathrm{H}^{3}$ without fixed points or, equivalently, that the projection

$$
\pi: \not \nvdash^{3} \rightarrow \not \not^{3} / G=M_{0}(G)
$$

is smooth. It then follows that $G$ is isomorphic to $\pi_{1} M_{0}(G) . \not^{3}$ may be modelled on the open unit disk $\Delta^{3} \subset \mathbf{R}^{3}$ with the metric

$$
d s^{2}=\left[4\left(1-|X|^{2}\right)\right]^{-2} d X^{2} .
$$

$\not^{3}$ has a natural boundary $\partial \mathscr{H}^{3}=\partial \Delta^{3}$ and a natural compactification as $\mathrm{Cl}\left(\Delta^{3}\right)$. It is elementary to show that $G$ acts conformally on $\partial \mathscr{H}^{3}$. There is a maximal open set $\Omega=\Omega(G) \subset \partial \Delta^{3}$ on which $G$ acts discontinuously. $\Omega(G)$ is called the set of discontinuity of $G . \Lambda(G)=\partial \mathcal{H}^{3} \backslash \Omega(G)$ is the limit set of $G$. The Kleinian manifold of $G$ is the bordered 3-manifold $M(G)=\left[\not^{3} \cup \Omega(G)\right] / G$.

Since $\not^{3}$ is contractible. $M(G)$ is irreducible, i.e. every 2-sphere $S^{2} \subset M(G)$ bounds an embedded 3-ball $B^{3} \subset M(G)$.

$G$ is said to be of the first kind if $\partial M(G)=\varnothing$; this occurs precisely when $\Omega(G)=\varnothing . G$ is of the second kind if $\partial M(G) \neq \varnothing$.

The elements $\gamma \in G$ act as Moebius transformations on $\partial \not^{3}$ and, as usual, are classified as loxodromic (including hyperbolic), parabolic or elliptic. Elliptic elements cannot occur in torsion-free groups since they have axes of rotation. If $\gamma$ is loxodromic then it has two fixed points on $\partial \mathscr{\gamma}^{3}$ and fixes the hyperbolic line connecting those endpoints.

If $H$ is a subgroup of $G$ and $A \subset \mathrm{Cl}\left(\not^{3}\right)$ then we say $A$ is precisely invariant under $H$ if $\eta(A)=A$ for $\eta \in H$ and $\eta(A) \cap A=\varnothing$ for $\eta \in G \backslash H$. It is classical that if $\gamma \in G$ is parabolic and has normalizer $N_{\gamma}$ then there is a 3 -ball $B^{3}$ which is precisely invariant under $N_{\gamma}$. It is easily seen that $G B^{3} / G=B^{3} / N_{\gamma}$ is topologically either $T^{2} \times[0,1)$ or $C \times[0,1]$ where $T^{2}$ is the 2 -torus and $C$ is an annulus or cylinder. In the latter case, Marden [6] has shown that up two solid cylinders $B^{i}$ may be adjoined to $B^{3}$, connecting $B^{3}$ to $\Omega(G)$, with precise invariance preserved. Removing these cylinders from $M(G)$ has the effect, topologically, of removing the punctures on $\partial M(G)$ and replacing them with border curves. If two such cylinders are present, the corresponding border curves are glued together and the parabolic singularity disappears. We denote by $M^{+}=M^{+}(G)$ the bordered 3-manifold obtained from $M(G)$ by removing the projections of the horoballs $\mathrm{Cl}\left(B^{3}\right)$ and those cylinders $B_{i}$ which connect $B^{3}$ to $\Omega(G)$ as above. The cylinders and tori which form $M \backslash M^{+}$ are called cusps. $\Omega^{+}(G)$ is $\Omega(G)$ with the $B_{i}$ removed.

A powerful, but seemingly neglected, theorem in the study of these manifolds has been provided by Peter Scott [8]. He showed that any 3 -manifold $M$ with finitely generated fundamental group contains a compact submanifold $i: N \hookrightarrow M$ so that $\pi_{1}(i)$ is an isomorphism. For hyperbolic manifolds, one may fill in any omitted balls to obtain a compact 3-submanifold $i: N \hookrightarrow M$ which is a homotopy equivalence. A compact submanifold $i$ : $N \hookrightarrow M$ which is a homotopy equivalence is called ample.

Let $N$ be an ample submanifold of $M=M(G)$ and, for each border component $S$ of $N$, let $M_{S}$ denote the component of $M \backslash N$ bounded by $S . N$ is called wellpositioned if, for each component $S$ of $\partial N$ and each loop $\alpha$ in $M_{S}$ which is nontrivial in both $M$ and $M_{S}, \alpha$ is freely homotopic in $M_{S}$ to a loop in $S$. Let $\mathcal{G}_{1}$ denote the class of torsion-free finitely generated Kleinian groups $G$ such that $M(G)$ has a well-positioned ample submanifold. If $G \in \mathcal{G}_{1}$ we shall say that $M(G) \in \mathcal{M}_{1}$. 


\section{Basic properties of 3-manifolds and well-positioned ample subman-} ifolds.

Proposition 1. Let $M$ be a 3-manifold and $S, S_{1}, S_{2}$ be three distinct incompressible components of $\partial M$. If $\alpha_{1}, \alpha_{2}$ are two nontrivial simple loops in $S_{1}, S_{2}$ respectively and satisfy:

(1) $\alpha_{1}$ and $\alpha_{2}$ are not homotopic in $M$, and

(2) $\alpha_{1}$ and $\alpha_{2}$ are homotopic to loops in $S$, then there are disjoint annuli $A_{1}$ and $A_{2}$ with $A_{i}$ joining a nontrivial simple loop on $S$ to $\alpha_{i}$.

Proof (PRIVATEly COMMUNiCATED By G. A. SWARUP). By Waldhausen's theorem [10], there exist annuli $A_{1}$ and $A_{2}$ connecting $\alpha_{1}$ and $\alpha_{2}$ respectively to $S$. $A_{i} \cap S$ is a simple loop and we must only show that the $A_{i}$ may be redefined so that they retain their defining properties and $A_{1} \cap A_{2}=\varnothing$. By simplicial approximation we can make $A_{1}$ and $A_{2}$ transverse and look at $A_{1} \cap A_{2}$ in $A_{1}$. The intersection will consist of a finite number of simple curves of three distinct types. They are either closed of have their endpoints on the border surfaces (see Figure 1). An intersection curve running from one border curve of $A_{1}$ to the other is ruled out by our hypothesis. The curves of intersection are disjoint by the transversality of the intersection and the fact that the two annuli are embedded. The curves of intersection are therefore nested and we will eliminate them starting at the innermost curve by modifying the embeddings.

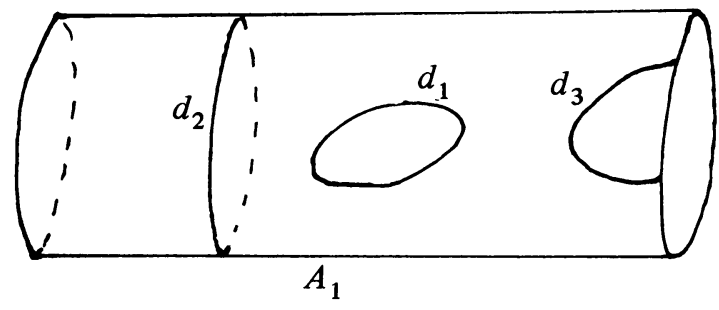

\section{FIGURE 1}

The $d_{1}$ curve. $d_{1}$ bounds a disk $D_{1}=D_{1}^{\prime}$ in $A_{1}$. Since $\pi_{1} A_{1} \hookrightarrow \pi_{1} M$ is injective, $d_{1}$ must also bound a disk $D_{2}$ in $A_{2}$. The latter disk may also contain intersection curves, so we replace it by a parallel copy $D_{2}^{\prime}$ of $D_{1}$. We then reduce the number of intersection curves setting $A_{i}^{\prime}=\left(A_{i} \backslash D_{i}\right) \cap D_{i}^{\prime}$ and then perturbing a bit and regluing. $A_{i}^{\prime}$ has a reduced number of components of the intersection.

The $d_{2}$ curve. Again since $\pi_{1} A_{i} \hookrightarrow \pi_{1} M$ is injective, $d_{2}$ is not trivial in $A_{2}$. It follows that $d_{2}, \alpha_{1}$ and $\alpha_{2}$ are homotopic in $M$, coitrary to hypothesis.

The $d_{3}$ curve. In this case $d_{3}$ bounds a disk $D_{1}$ in $A_{1}$ hence also a disk $D_{2}$ in $A_{2}$. Continue as for the $d_{1}$ curve.

Since there are only finitely many components of $A_{1} \cap A_{2}$, we can remove them all.

Proposition 2. Let $M \in \mathcal{M}_{1}$ and $S^{\prime}$ be a border component of $M$. Further assume that $\alpha_{1}$ and $\alpha_{2}$ are simple homotopically nontrivial curves on $S^{\prime}$ which intersect transversally at either 1 or 2 points. If $N$ is a well-positioned ample submanifold of $M$ and $S$ is a border component of $N$ with $S^{\prime} \subset M_{S}$, then there 
exist annuli $A_{i}$ connecting $\alpha_{i}$ to loops on $S$ such that the $A_{i}$ intersect in the same number of arcs as the $\alpha_{i}$ intersect in points.

ProOF. Since $N$ is a well-positioned ample submanifold of $M$, we see immediately that the inclusion map induces an isomorphism of $\pi_{1} S$ and $\pi_{1} M_{S}$. Thus there exist immersed annuli $A_{i}$ connecting $\alpha_{i}$ to a loop $\alpha_{i}^{\prime}$ lying on $S$. If the annuli are embedded, as we may assume from Waldhausen's theorem applied to $M_{S}$, then the techniques of the previous proposition may be used to remove all arcs of intersection which do not run from one border curve of each annulus to the other border curve. Further the same techniques imply that these arcs may be taken disjoint except for endpoints. The proposition then reduces to showing that the embedding of the annuli $A_{i}$ may be chosen so that an intersection point of the loops $\alpha_{i}$ lies on exactly one curve of intersection of the $A_{i}$.

When two arcs of intersection have the same endpoints, together they bound disks in both annuli and both intersections can be removed as in the case of the $d_{1}$ curve of the previous proposition. Suppose there are two simple $\operatorname{arcs} \beta_{i} \subset A_{1} \cap A_{2}$ which connect the border components of the $A_{i}$ and share only one endpoint. Then there are $\operatorname{arcs} \varepsilon_{i} \subset A_{i} \cap S$ so that $\beta_{1} \cup \beta_{2} \cup \varepsilon_{i}$ bounds a triangle $T_{i}$ in $A_{i} . T_{1} \cup T_{2}$ is an immersed disk in $M_{S}$ bounded by $\beta=\varepsilon_{1} \cup \varepsilon_{2}$. Thus $\beta$ is null-homotopic in $M_{S}$ and bounds an embedded disk $D_{1}$ in $M_{S}$. As mentioned at the beginning of the proof, $\pi_{1} M_{S}$ and $\pi_{1} S$ are canonically isomorphic, so $\beta$ is trivial in $S$ and bounds a disk there. A homotopy of $\beta$ to a point in $S$ deforms the curves $\beta_{i}$ to curves with two common endpoints - the case which was previously considered. Thus in all cases $A_{1} \cap A_{2} \cap S$ has the same number of points as $A_{1} \cap A_{2} \cap S^{\prime}$.

The assumption that a hyperbolic manifold has a well-positioned ample submanifold has one significant consequence. The next proposition shows that the assumption leads to a compact submanifold that carries the homotopy of the boundary of the ambient manifold.

PROPOSITION 3. If $M \in \mathcal{M}_{1}$, then there exists an ample submanifold $N$ of $M$ so that the border of $N$ consists of all points of the border of $M$ except for small neighborhoods of the punctures.

ProOF. Let $S_{1}$ be any border component of $M$. From $S_{1}$ delete a small neighborhood of each of the punctures and call the resulting surface $S_{1}^{\prime}$. Using simple loops and arcs from these loops to the border curves of $S_{1}^{\prime}, S_{1}^{\prime}$ can be carved up into simply connected pieces. We may further assume that the arcs and simple loops pairwise intersect in at most two points. Using the previous propositions, we may find corresponding annuli connecting the loops on $S_{1}^{\prime}$ to a single border component $S$ of $N$. By deforming the annuli sequentially in pairs, we may assume that the annuli pairwise intersect along arcs connecting $S$ to $S_{1}^{\prime}$. Since $M$ is irreducible and $S \cup S_{1}^{\prime} \cup\{$ the annuli $\}$ consists of a collection of 2-spheres, we may assume that the annuli bound solid cylinders. $N$, together with these cylinders, is compact and has $S_{1}^{\prime}$ in its border.

The previous argument is rather terse, but is completely standard. It is the foundation of Waldhausen's theory of sufficiently large 3-manifolds [11]. 
3. The deficiency index. In his paper on the Euler characteristics of groups, Wall [12] showed that an abstract group $\Gamma$ has a well-defined Euler characteristic $\chi(\Gamma)$, and when $\Gamma$ is the fundamental group of a manifold $M, \chi(\Gamma)=\chi(M)$. An immediate consequence is

LEMMA 1. If $N$ and $N^{\prime}$ are ample submanifolds of the Kleinian manifold $M(G)$ then $\chi(N)=\chi\left(N^{\prime}\right)$.

In genus $g \geq 1$ we may speak of the hyperbolic area of a compact connected surface $S$. It is precisely $A(S)=-2 \pi \chi(S)$. Notice that tori are assigned hyperbolic area zero. If $S$ is a punctured connected surface of type $(g, n)$, its hyperbolic area is $2 \pi(2 g-2+n)$. If horocyclic neighborhoods $N_{i}$ of two punctures are deleted and we identify $\partial N_{1}$ to $\partial N_{2}$, the hyperbolic area remains unchanged (see Abikoff [1] for a discussion of intrinsic metrics on Riemann surfaces).

Since a Kleinian manifold $M(G)$ with $G$ nonelementary is aspherical. $A(\partial M)$ is well defined as are $A\left(\partial M^{+}\right)$and $A(\partial N)$ for any ample submanifold $N$; we simply add up the hyperbolic areas of the (nontoral) components of the boundary.

DEFINITION. If $M=M(G) \in \mathcal{M}_{1}$ is a Kleinian manifold with ample submanifold $N$, the deficiency index of $M$ (or $G$ ) is $\delta(M)=\delta(G)=A(\partial N)-A(\partial M)$.

That $\delta(M)$ measures the area of the part of $\partial N$ which has been "degenerated" in $M$ is shown by

THEOREM 1. If $G \in \mathcal{G}_{1}$ then $\delta(G)=\delta(M(G)) \geq 0$ with equality if and only if $G$ is geometrically finite.

ProOF. $A(\partial N)=-2 \pi \chi(\partial N)$ which is independent of the choice of $N$. Since $M \in \mathcal{M}_{1}$, we may choose $N$ so that $\partial N \supset \partial M^{+}$. But $A\left(\partial M^{+}\right)=A(\partial M)$ and it follows that $A(\partial N) \geq A(\partial M)$ with equality if and only if $\partial N=\partial M^{+}$.

If $M^{+}$is compact then $M^{+}$is ample. Thus a geometrically finite group has deficiency zero. It is trivial that $M$ is geometrically finite when $M^{+}$is ample.

4. The area theorems and number of components of the boundary. Classical results in Kleinian groups theory (see Bers [3]) have shown that $A(\partial M(G)) \leq 4 \pi(\nu-1)$ where $\nu$ is the rank of $\pi_{1} M(G)$. The usual proof uses cohomological machinery. In the past, Bers [4] and Maskit [7] were able to use area inequalities to study whether or not a $b$-group is degenerate. That area theorems express degeneracy for all Kleinian manifolds with well-positioned ample submanifolds is a direct consequence of Theorem 1 and some elementary topology. Let $G \in \mathcal{G}_{1}, \nu$ be the rank of $G$ and $\beta_{i}$ be the $i$ th Betti number of some ample submanifold $N$ of $M(G)$. Since $N$ is a compact bordered 3-manifold, elementary odd dimensional topology shows that $\chi(\partial N)=2 \chi(N)$. Thus by Theorem 1 ,

$$
A(\partial M) \leq A(\partial N)=-4 \pi \chi(N)=4 \pi\left[\beta_{1}-1-\beta_{2}\right]
$$

because $\beta_{0}=1$ and $\beta_{3}=0$ for the compact bordered manifold $N$. Since

$$
\beta_{1}=\operatorname{rank} \text { of } H_{1}(N) \leq \operatorname{rank} \pi_{1} N=\nu,
$$

we have

THEOREM 2. If $M \in \mathcal{M}_{1}$ and $N$ is a well-positioned ample submanifold of $M$ then

$$
A(\partial M) \leq A(\partial N) \leq 4 \pi\left[\nu-1-\beta_{2}\right] \leq 4 \pi[\nu-1]
$$


Equality holds in the first inequality if and only if

(i) $G$ is geometrically finite.

Equality holds in the second inequality if and only if

(ii) $\operatorname{rank} H_{1}(M)=\operatorname{rank} \pi_{1} M$.

Equality holds in the last inequality if and only if

(iii) $\beta_{2}=0$.

$\beta_{2}$ measures the number of connected incompressible surfaces in $N$ which are not parallel to all of the boundary simultaneously. If $\partial N$ is not connected then $A(\partial M)<4 \pi[\nu-1]$. As an example take a Fuchsian group $G$ representing a compact surface. $G$ is geometrically finite, $\operatorname{rank} H_{1}=\operatorname{rank} \pi_{1}$ but $\beta_{1}=1$, so $A\left(\partial M^{+}\right)=4 \pi[\nu-2] \cdot A\left(\partial M^{+}\right)=4 \pi[\nu-1]$ for Fuchsian groups of the second kind and Fuchsian groups of the first kind with parabolic elements.

This technique also yields a sharp bound on the number of components of $\partial M$. Since each component of $\partial M$ must have area $\geq 2 \pi$, we have

THEOREM 3. If $M \in \mathcal{M}_{1}$, the number $\nu_{k}$ of components of $\partial M$ satisfies

$$
\begin{aligned}
\nu_{k} & \leq A(\partial M) / 2 \pi \\
& \leq 2\left[\nu-1-\beta_{2}\right] \\
& \leq 2[\nu-1] .
\end{aligned}
$$

Equality holds in (4.2) if and only if

(i) $G$ is geometrically finite,

(ii) $\operatorname{rank} H_{1} M=\operatorname{rank} \pi_{1} M$, and

(iii) each component of $M$ is a thrice punctured sphere.

Equality holds in (4.3) if and only if (i)-(iv) hold, where

(iv) $\beta_{2}=0$.

Equality in (4.3) holds for a Fuchsian group representing a thrice punctured sphere. Inequality (4.3) had previously been obtained by Marden [6], refining the results of Ahlfors [2] and Bers [3].

5. Parabolic conjugacy classes. Ahlfors was first to raise the question of whether the number of cusps or, equivalently, the number of primary parabolic conjugacy classes in a finitely generated Kleinian group $G$ is finite. For geometrically finite groups the result is obvious; for geometrically infinite groups the result has been asserted by Kulkarni and Thurston and has been proved by Sullivan [9] (see also Kra's addenda to the proof [5]). Here we prove the result for $G \in \mathcal{G}_{1}$ as an elementary consequence of Proposition 1 and Theorem 3 and get a sharp estimate for the number $N_{C}$ of cusps or, equivalently, conjugacy classes of maximal parabolic subgroups, in torsion-free groups in $\mathcal{G}_{1}$. These groups are either cyclic, in which case they correspond to cusp cylinders in $G$, or free abelian of rank two, corresponding to cusp tori. Let $N_{T}$ be the number of cusp tori and $N_{A}$ be the number of cusp cylinders for $G$. $N_{C}$ is then the sum of $N_{T}$ and $N_{A}$.

THEOREM 4. If $G \in \mathcal{G}_{1}$ is a nonelementary finitely generated torsion-free Kleinian group on $\nu$ generators and $N$ is a well-positioned ample submanifold of 
$M(G)$ as in Proposition 3, then

$$
\begin{gathered}
N_{A} \leq 3\left[\nu-1-\beta_{2}\right] \\
N_{T} \leq \beta_{2}+1, \\
N_{C} \leq 3 \nu-3 .
\end{gathered}
$$

Equality holds in (5.1) if and only if $G$ is geometrically finite and each component of $\partial M(G)$ is a thrice punctured sphere. Equality holds in (5.2) if and only if $G$ is geometrically finite of the first kind and $\mathrm{H}_{2}(M)$ is generated by the components of $\partial M^{+}$. Equality holds in (5.3) if and only if $G$ is geometrically finite, each component of $\partial M(G)$ is a thrice punctured sphere, $\partial M^{+}(G)$ is connected, $\beta_{2}=0$ and $\operatorname{rank} \pi_{1} M(G)=\operatorname{rank} H_{1}(M(G))$.

ProOF. Each cusped cylinder in $M(G)$ determines a simple closed curve in $\partial N$. These curves are disjoint and homotopically independent. The maximal possible number of cusped cylinders occurs when each nontoral surface in $\partial N$ is dissected by these curves into triply connected domains. The count of the number of cusp tori is not affected by whether or not a simple closed curve in $\partial N$ is associated to a cusp cylinder in $M$. Thus we maximize the number of cusp cylinders, hence also $N_{C}$, by assuming that each nontoral component of $\partial N$ has been maximally dissected by homotopically independent, disjoint simple closed curves. Equivalently, we are assuming that each nontoral component of $\partial N$ is carved up into triply-connected domains. Each triply-connected domain contributes $2 \pi$ to $A(\partial N)$ and corresponds to $3 / 2$ cusps. If $\nu_{k}^{\prime}$ is the number of triply connected domains forming the nontoral part of $\partial N$,

$$
N_{A} \leq(3 / 2) \nu_{k}^{\prime} \leq(3 / 2) A(\partial N) / 2 \pi \leq 3\left[\nu-1-\beta_{2}\right] .
$$

For equality to occur, each triply connected domain must correspond to a thrice punctured sphere. A thrice punctured sphere can never be degenerate so $\delta(G)=0$ and $G$ is geometrically finite. Further, $\nu=\operatorname{rank} \pi_{1} N=\operatorname{rank} H_{1}(N)$. The converse is trivial and (5.1) is proved.

Each cusped torus determines a unique boundary component of $N . \partial N$ bounds in $N$. However, if $N$ has more than one component, all but one component of $\partial N$ are free generators of $H_{2}(N)$. Thus $N_{T} \leq \beta_{2}+1$. If $\partial N$ consists solely of tori then $\delta(G)=0$ and $G$ is geometrically finite of the first kind. For equality to hold in (5.2), the tori in $\partial(N)$ must generate $H_{2}(N)$. Conversely, if equality holds, $\partial N$ must have only toral components. Thus $\delta(G)=0$ and $G$ is geometrically finite. Further, the components of $\partial N$ generate $\mathrm{H}_{2}\left(M^{+}\right)$.

The above argument shows that $\beta_{2} \geq N_{T}-1$ if $\partial M(G)=\varnothing$ and $\beta_{2} \geq N_{T}$ if $\partial M(G) \neq \varnothing$. Using (5.1) we then obtain

$$
N_{A}+\beta_{2} \leq N_{A}+3 \beta_{2} \leq 3[\nu-1] .
$$

Together, these inequalities give

$$
3[\nu-1] \geq \begin{cases}N_{A}+N_{T} & \text { if } \partial M(G) \neq \varnothing, \\ N_{A}+N_{T}-1 & \text { if } \partial M(G)=\varnothing .\end{cases}
$$

We have thus shown

$$
N_{C} \leq \begin{cases}3[\nu-1] & \text { if } \partial M(G) \neq \varnothing \\ 3 \nu-2] & \text { if } \partial M(G)=\varnothing .\end{cases}
$$


To prove (5.3) we must rule out the case that $N_{C}=3 \nu-2$. If so, (5.4) is an equality; hence $\beta_{2}=3 \beta_{2}$ or $\beta_{2}=0$. By (5.5), $\partial M(G)=\varnothing$; hence $N_{A}=0$ and $N_{C}=1$. If $\nu=1$, then $G$ is elementary contrary to hypothesis. Any other equality is impossible. Thus (5.3) is valid. To have equality in (5.3), we must have equality in (5.4). Thus $\beta_{2}=0$ and $G$ must be geometrically finite with at most one boundary component to $M^{+}(G)$. To obtain (5.4) as an equality we must have $\operatorname{rank} \pi_{1} M(G)=\operatorname{rank} H_{1}(M(G))$. As above, $N_{T}=0$; hence $N_{C}=N_{A}$ and, from equality in (5.1), we know that each component of $\partial M(G)$ must be a thrice punctured sphere.

A Fuchsian group $G$ representing a thrice punctured sphere shows that inequality (5.3) is sharp. $M^{+}(G)$ is then a handlebody of genus 2 , so $\beta_{2}=0 . \pi_{1} M(G)$ is free, so $\operatorname{rank} \pi_{1} M(G)=\operatorname{rank} H_{1}(M(G))$. Further, $G$ is geometrically finite. $G$ has 2 generators and 3 cusps.

The methods of the previous proof allow a sharp estimate for the number $N_{P}=$ $N_{A}+2 N_{T}$ of conjugacy classes of "linearly independent" maximal cyclic parabolic subgroups.

Corollary. $N_{P} \leq 3[\nu-1]$ unless $\beta_{2}=1, N_{T}=2, N_{A}=0, \nu=2$ and $G$ is geometrically finite.

ProOF. If $N_{A} \neq 0$, use (5.4) to obtain

$$
N_{A}+2 N_{T} \leq N_{A}+2 \beta_{2} \leq N_{A}+3 \beta_{2} \leq 3[\nu-1] .
$$

If $N_{A}=0,(5.4)$ yields

$$
2\left(N_{T}-1\right)+\beta_{2} \leq 3 \beta_{2} \leq 3[\nu-1] .
$$

When $\beta_{2} \geq 2$, the desired result is valid. If $\beta_{2}=0$, then $N_{T}$ is either 0 or 1 , which is clearly dominated by $3 \leq 3[\nu-1]$ for a nonelementary group. If $\beta_{2}=1$, then $2 N_{T}=0,2$ or 4 , and the result is valid unless $N_{T}=2, \nu=2$ and $G$ is geometrically finite.

John Ratcliffe has observed that the Whitehead link complement satisfies $N_{P}=$ $3 \nu-2$ and is therefore an example of the exceptional case.

6. The existence of well-positioned ample submanifolds. It has been conjectured that the quotient manifold of every torsion-free finitely generated Kleinian group is, but for neighborhoods of cusps, the interior of a compact manifold. If so, our main assumption, namely, the existence of a well-positioned ample submanifold, is clearly valid. Using Marden's Basic Structure Theorem [6, p. 426] or directly from Waldhausen's theory, existence has been proved for function groups and a class of groups satisfying a complicated topological assumption. We have already shown that the assumption is valid for geometrically finite groups. More interesting is that the assumption is valid for groups which have only trivial free product decompositions. This last result follows directly from Swarup's original argument which Scott extended to prove the existence of ample submanifolds. More generally, one can show that the assumption is valid if the free product decompositions can be realized geometrically, in the sense of the Maskit decomposition theorems, along closed disks. 


\section{REFERENCES}

1. W. Abikoff, The real analytic theory of Teichmuller space, Lecture Notes in Math., vol. 820, Springer-Verlag, Berlin and New York, 1980.

2. L. Ahlfors, Eichler integrals and the area theorem of Bers, Michigan Math. J. 15 (1968), 257-263.

3. L. Bers, Inequalities for finitely generated Kleinian groups, J. Analyse Math. 18 (1967), $23-41$.

4. (1970), 570-600.

5. I. Kra, On cohomology of Kleinian groups. IV, The Ahlfors-Sullivan construction of holomorphic Eichler integrals, J. Analyse Math. 43 (1983/4), 51-87.

6. A. Marden, The geometry of finitely generated Kleinian groups, Ann. of Math. 99 (1974), 383-462.

7. B. Maskit, On boundaries of Teichmuller spaces and on Kleinian groups, Ann. of Math. 91 (1970), 607-639.

8. G. P. Scott, Compact submanifolds of 3-manifolds, J. London Math. Soc. (2) 7 (1973), 246-250.

9. D. Sullivan, A finiteness theorem for cusps, Acta Math. 147 (1981), 289-299.

10. F. Waldhausen, Eine Verallgemeinerung des Schleifensatzes, Topology 6 (1967), 501-504.

11. __ On irreducible 3-manifolds which are sufficiently large, Ann. of Math. 87 (1968), $56-88$.

12. C. T. C. Wall, Rational Euler characteristics, Proc. Cambridge Philos. Soc. 57 (1961), 182-183.

Department of Mathematics, University of Connecticut, StorRs, ConNECTICUT 06862 